\title{
Noise and signal estimation in MRI: two-parametric analysis of rice-distributed data by means of the maximum likelihood approach
}

\author{
Tatiana V. Yakovleva, Nicolas S. Kulberg \\ Department of Algorithm Theory and Coding Mathematical Principles, Institution of Russian Academy of Sciences Dorodnicyn \\ Computing Centre of RAS, Moscow, Russia
}

\section{Email address:}

tan-ya@bk.ru(T. V. Yakovleva), kulberg@yandex.ru(N. S. Kulberg)

\section{To cite this article:}

Tatiana V. Yakovleva, Nicolas S. Kulberg. Noise and Signal Estimation in MRI: Two-Parametric Analysis of Rice-Distributed Data by Means of the Maximum Likelihood Approach, American Journal of Theoretical and Applied Statistics. Vol. 2, No. 3, 2013 , pp. 67-79. doi: $10.11648 /$ j.ajtas.20130203.15

\begin{abstract}
The paper's subject is the elaboration of a new approach to image analysis on the basis of the maximum likelihood method. This approach allows to get simultaneous estimation of both the image noise and the signal within the Rician statistical model. An essential novelty and advantage of the proposed approach consists in reducing the task of solving the system of two nonlinear equations for two unknown variables to the task of calculating one variable on the basis of one equation. Solving this task is important in particular for the purposes of the magnetic-resonance images processing as well as for mining the data from any kind of images on the basis of the signal's envelope analysis. The peculiarity of the consideration presented in this paper consists in the possibility to apply the developed theoretical technique for noise suppression algorithms' elaboration by means of calculating not only the signal mean value but the value of the Rice distributed signal's dispersion, as well. From the view point of the computational cost the procedure of the both parameters' estimation by proposed technique has appeared to be not more complicated than one-parametric optimization. The present paper is accented upon the deep theoretical analysis of the maximum likelihood method for the two-parametric task in the Rician distributed image processing. As the maximum likelihood method is known to be the most precise, its developed two-parametric version can be considered both as a new effective tool to process the Rician images and as a good facility to evaluate the precision of other two-parametric techniques by means of their comparing with the technique proposed in the present paper.
\end{abstract}

Keywords: Rice Distribution, Maximum Likelihood Method, MR Imaging, Two-Parametric Analysis

\section{Introduction}

The problem of the image noise suppression may be considered as a special case of the problem of the unknown statistical parameters estimation within the frameworks of any statistical model on the basis of the measured data. To obtain the correct estimation of the parameters it is important to use an adequate statistical model describing the corresponding physical process.

In many tasks connected with the visualization the noise is formed by means of summing a big number of independent components that distort the initial image signal. Thus such a noise obeys the Gaussian distribution. The same mechanism works at forming the noise distorting the real and imaginary parts of the image signal in the systems of magnetic-resonance visualization [1]. However normally at MR image formation the value to be analyzed is the amplitude of the signal instead its real and imaginary parts. This amplitude obeys the Rice statistical distribution $[2,3,4]$. The applicability of this statistical model for describing the MR visualization has been proved in many works (for example, $[3,5,6]$ ).

The Rice distribution characterizes amplitude of the stochastic signal as a square root of the sum of squares of two stochastic values while each of these values obeys the Gaussian statistics. Unlike the noise of Gaussian (normal) distribution the Rician distributed noise is not an additive one. An important peculiarity of such a noise consists in the fact that this noise does not only adds the stochastic distortions into the data contained in the image, but also creates a background depending upon the value of the 
signal. Such a background leads to the decrease of the image contrast, especially at low values of the signal-to-noise ratio.

The noise influence within frameworks of the applicability of the Rice statistical model depends upon the signal value. That's why the mathematical methods describing the processes that obey to the Rice distribution as well as the corresponding transformations of the obtained signal with the purpose of the noise suppression at the image formation and processing are essentially nonlinear. The strict mathematical description of such a noise is rather a complicated mathematical task. Two approximate limiting cases are known that are used for the construction of the simplified analysis schemes: at low signal-to-noise ratio the Rice distribution is transformed into the Rayleigh distribution, while at high signal-to-noise ratio - into the normal Gaussian distribution.

It is worth to note that many authors use the linear methods for analyzing the magnetic-resonance images, although these methods have been developed first of all for the data obeying the Gaussian distribution, (see, for example, [7, 8,9]). However this model describes the process correct only at very high values of the signal-to-noise ratio, while in other cases the Rice distribution differs from the Gaussian one significantly. In these situations applying the linear methods leads to a bias of the data obtained as a result of such an analysis if compared with the real data.

To escape the appearance of such a bias and to obtain the more correct values of the parameters at arbitrary value of the signal-to-noise ratio the nonlinear techniques are used more often for the magnetic resonance images filtration in the papers of the last years. These techniques are based upon the application of the Rice statistical model, [10, 11, $12,13,14,15]$. In all these papers the maximum likelihood (further-ML) method is used for the estimation of the parameter of a signal mean value, which is undoubtedly important but not the single and completely sufficient parameter that allows reconstructing an image. The second meaningful statistical parameter of the task - a dispersion of the data forming an image - is supposed to be known in the mentioned papers. The value of dispersion is often measured by means of the empirical techniques which are not based upon the Rice statistical model. Paper [14] is worth to be mentioned as providing a comparative analysis of the ML and the mean-square error (MSE) methods for the statistical parameters' estimation based upon the Kramer-Rao lower bound. The papers are known (for example, [16]), in which the noise is measured within the Rice model although based not upon the ML method, but upon the simplified scheme that could be considered as not optimal one, namely - the mean-square error minimization.

In the present paper the ML method is first used for the estimation of both a priori unknown statistical parameters the image signal mean value and the dispersion. The correct estimation of these parameters makes it possible to solve the problem of noise suppression and the image reconstruction in the magnetic-resonance visualization systems much more efficiently.

After finishing the work that has become the subject of the present paper we learned about the paper [17] which considers the properties of the maximum likelihood equations' solutions and their quantity.

In spite of the thematic affinity between paper [17] and some issues of the present paper relating the properties of solutions of the maximum likelihood equations the approaches and the apparatus of mathematical analysis developed in the present paper and in paper [17] are essentially different. We suppose that it is reasonable to conduct here a comparative analysis of the both papers' approaches and results. While considering the one-parametric task, i.e. the task of the estimation of only one unknown parameter - the amplitude of the initial signal - while a dispersion value is supposed to be known a priory, we have implemented a detailed mathematical analysis of the extremum's character of the likelihood function at the points of zero value of its derivative by means of the strict mathematical proof and study of the function's features. These features which visually seem evidently following from the function's graph need nevertheless to be strictly proved what has been done in the present paper by means of a number of lemmas and theorems. As for the paper [17] it has not provided the complete mathematical proof of the function's features which determine the existence of the task solution: the related functions' features and the conclusion on the existence of the task solution are declared on the basis of graphical illustration.

In the present paper we have complimented a comprehensive mathematical investigation of the functions determining the character and the properties of the maximum likelihood equation's solution as for the one-parametric and for two-parametric tasks.

It is important that at solving the two-parametric task, when the maximum likelihood technique is applied to find the both unknown statistical parameters - the initial signal's mathematical expectation and the noise dispersion - we managed to reduce solving the system of two nonlinear equation with two variables to solving one equation with one variable.

Thus the proposed by us method of proving the properties of the maximum likelihood equation's solution differs principally from the purely graphical approach presented in paper [17], which although contains the analysis of the second derivatives of the likelihood function, but does not provide the strict proof of the properties (the monotonous character, the smoothness, the convexity/concavity etc.) of the functions which determine the conditions of the task solutions existence and their quantity. 


\section{The Problem Formulation. the Maximum Likelihood Equations System}

At constructing the magnetic-resonance image a value being measured is a modulus of a complex value with the real and imaginary parts being distorted by the Gaussian noise. This noise obeys a normal distribution. The mean values of the noise components distorting the measured signals' real and imaginary parts are obviously of zero value, while the value of the noise dispersion is of an a-priory unknown value.

Let us denote by $x_{\mathrm{Re}}$ and $x_{\mathrm{Im}}$ the independent random quantities having the normal distribution with the same dispersions and non-zero mean values. These quantities correspond to the real and imaginary parts of the complex signal

$$
x=\sqrt{x_{\mathrm{Re}}^{2}+x_{\mathrm{Im}}^{2}}
$$

forming the magnetic-resonance image under the study. Let us denote as $v$ the mean value of the real and imaginary parts of the measured signal; as $\sigma^{2}$ - the dispersion of the Gaussian noise distorting the signal. Then the amplitude $x$ of the signal obeys the Rice distribution with the following probability density function:

$$
P(x \mid v, \sigma)=\frac{x}{\sigma^{2}} \cdot \exp \left(-\frac{x^{2}+v^{2}}{2 \sigma^{2}}\right) \cdot I_{0}\left(\frac{x v}{\sigma^{2}}\right)
$$

Here and below we use the following designations:

$$
I_{\alpha}(z) \text { is the modified Bessel function (Infeld function) }
$$
of the first kind with order $\alpha, x_{i}$ is the signal value measured in $i$-th sample for the subsequent image data processing; $n$ is the quantity of the elements in the sample (the so-called sample's length). For designating the averaging within the sample we'll use the angular brackets:

$$
\langle x\rangle=\frac{1}{n} \sum_{i=1}^{n} x_{i},\left\langle x^{2}\right\rangle=\frac{1}{n} \sum_{i=1}^{n} x_{i}^{2}
$$

The mathematical problem being solved in the present paper consists in the estimation of the both mentioned parameters $v$ and $\sigma^{2}$ on the basis of the measured sample data and in the further reconstruction of the initial undistorted image.

To solve this problem we apply the ML method being widely used in similar tasks [18, 19], especially in magnetic-resonance imaging that has become one of the most efficient instrument in medical diagnostics, [20-22]. Applied to the problem of the magnetic-resonance images processing the ML method has been used in many papers (for example, $[10,11,12,13,14,15]$ ) for the estimation of one among several unknown parameters of the task, namely- the parameter of the mean value $v$ of a signal, forming the undistorted image. In these papers the second parameter - dispersion - is supposed to be known although normally this is not the case in practice. Some authors propose to measure the value of dispersion taking the data from the areas of an image with very low signal level, on the basis of the noise background or from the areas with high signal-to-noise ratio having locally-constant signal level [3]. However such calculations do lead to a noticeable systematic error in the estimation of a dispersion value and that's why cannot be considered as reliable. The error in computing the dispersion inevitably causes an error in the useful signal estimation. So the task of the accurate evaluation of the dispersion value $\sigma$ is rather actual for the subsequent accurate estimation of the parameter $v$.

An important point is that in contrast to the previous papers devoted to the problem of the magnetic-resonance visualization, in particular the papers $[11,14]$ considering the maximum likelihood approach in its one-parametric approximation, the present paper is the first to develop the two-parametric version for the maximum likelihood technique applied for the magnetic-resonance vision tasks.

Let us consider a sample of $n$ measurements of the value of the signal's amplitude $x$. The function of the joint probability density $L(v, \sigma)$ of the events consisting in the fact that the result of the $i$-th measurement equals to the value $x_{i}(i=1, \ldots, n)$ can be expressed as a product of the probability density functions for each measurement of the sample:

$$
L(v, \sigma)=\prod_{i=1}^{n} P\left(x_{i} \mid v, \sigma\right)
$$

where the function $P\left(x_{i} \mid v, \sigma\right)$ is determined by the expression (1). The function is also referred to as the likelihood function (further we'll denote it shortly as LF). At the known samples' data having been obtained as a result of the measurements this function depends upon unknown statistical parameters $v$ and $\sigma^{2}$. The ML method consists in the finding the parameters' values which maximize LF (or, equivalently, its logarithm). The logarithmic likelihood function (LLF) for the Rice distribution is as follows:

$$
\begin{aligned}
& \ln L(v, \sigma)=\sum_{i=1}^{n} \ln P\left(x_{i} \mid v, \sigma\right) \\
& =\sum_{i=1}^{n}\left\{-2 \cdot \ln \sigma-\frac{x_{i}^{2}+v^{2}}{2 \cdot \sigma^{2}}+\ln I_{0}\left(\frac{x_{i} v}{\sigma^{2}}\right)\right\}
\end{aligned}
$$

The formula (3) is obtained from the formulas (2) and (1). In the expression for the LLF the terms are missed that do not depend upon the parameters to be evaluated as these terms do not influence upon the solution of the likelihood equations. The likelihood equations for computing the unknown statistical parameters $v$ and $\sigma^{2}$ is as follows: 


$$
\left\{\begin{array}{l}
\frac{\partial}{\partial v} \ln L(v, \sigma)=0 \\
\frac{\partial}{\partial \sigma} \ln L(v, \sigma)=0
\end{array}\right.
$$

The equating to zero the LLF derivatives allows finding those values of the parameters which provide an extremum value (maximum or minimum) of the LF. As the analytical solution of the equations (4) has not been found the task should be solved numerically. Solving the system of equation (4) with two unknown parameters involves the following evident difficulties, which have been resolved at the present paper:

1 . The determining of the conditions of the ML equations' solution existence and uniqueness is a cumbersome mathematical task;

2. Theoretical estimation of the found extremum points character (as that may correspond to both maximum and minimum of the LF) is also complicated;

3. The computational cost of the two-parametric optimization is by default higher than computational cost of the one-parametric task.

\section{One-Parametric ML Task}

For completeness and logical consistency of the theoretical consideration, before discussing the solution of the equations' system (4) for two unknown parameters $v$ and $\sigma^{2}$, we repeat in brief the solution of the task of finding only one parameter $v$ in supposition that $\sigma^{2}$ is known a priori. Just this case has become a subject for study in most of the mentioned above papers devoted to the Rice distributed data analysis by the ML method. Then we shall generalize the theoretical results for the case of evaluation of two unknown statistical parameters. Such an order of the material presentation will allow to follow the logic of the consideration more clearly. Then we'll consequently substantiate, by means of a number of lemmas and theorems, the existence and the uniqueness of the mathematical task solution obtained by the ML method.

Let us suppose that as a result of the measurements a sample of the signal values $x_{1}, \ldots x_{n}$ has been obtained. From formulas (3) and (4) it follows that in this case the first likelihood equation can be presented as follows:

$$
\frac{1}{n} \sum_{i=1}^{n} \frac{\partial}{\partial v} \ln I_{0}\left(\frac{x_{i} v}{\sigma^{2}}\right)-\frac{v}{\sigma^{2}}=0
$$

where $n$ is a number of the elements in the sample. Taking into account the following known expression [20]:

$$
\frac{d}{d z} I_{0}(z)=I_{1}(z)
$$

we can easily find the derivative within the sum sign in (5). This function and its properties will be important in the further consideration, so we shall introduce a special designation for it:

$$
\tilde{I}(z)=\frac{d}{d z} \ln I_{0}(z)=\frac{I_{1}(z)}{I_{0}(z)}
$$

Taking into account (7) the likelihood equation for the parameter $v$ can be written in as follows:

$$
v=\frac{1}{n} \sum_{i=1}^{n} \tilde{I}\left(\frac{x_{i} v}{\sigma^{2}}\right) \cdot x_{i}
$$

From (8) one can see that the properties of the solution of this equation are determined by the properties of the function $\tilde{I}(z)$ introduced by us in (7) and being equal to the ratio of the modified Bessel function of the first kind of the first and zero orders. The study of the properties of this function will help to consider the issue on the existence of the solutions of the ML equation (8), their quantity and features.

It is easy seen that the value $v=0$ is always one of the solutions of (8). We have proved the following statement (the proofs of this theorem and other mathematical statements are provided in the Appendix at the end of the paper):

Theorem 1

Let the condition $\left\langle x^{2}\right\rangle>2 \sigma^{2}$ be valid. Then at $v>0$ there exists a single solution of the equation (8) that corresponds to the maximum of the LLF. If $\left\langle x^{2}\right\rangle \leq 2 \sigma^{2}$, then the LLF maximum corresponds to the trivial solution of the equation (8), i.e. to the solution $v=0$.

Obviously while proving the theorem concerning the maximum likelihood technique we have to consider both the first and the second derivatives of the likelihood function in order to determine the character of the extremum (maximum or minimum) of the function in the point of zero first derivative. Similarly, the sign of the second derivative of the function being analyzed is taken into account in the mathematical considerations at proving Theorem 2 and Theorem 3 provided below.

The zero root of (8) corresponds to the LLF maximum only in the limiting case of the Rice distribution at $v=0$ (when it degenerates into the Rayleigh distribution). Such a distribution is characteristic for the noise component of the signal when the useful signal in the measured data is absent. In all other cases the zero root corresponds to the minimum of the LF.

It is worthwhile to note that the issue on the existence of the solution of the LLF maximum equation was being considered in detail in the paper [12]. The analysis of the LLF extremums was being conducted in this paper by means of the decomposition into the Taylor row taking into account some statements of the catastrophe theory concerning the possible structural changes of a function within the vicinity of the degenerate stationary point. In this paper the power expansion of the logarithmic likelihood function by the 
parameter $v$ is conducted, i.e. the decomposition within the vicinity of zero point $v=0$. In contrast to the way of consideration implemented by the authors of the paper [12] we study the behavior of the LLF with the purpose of the revealing all the solutions of the ML equation not limited by the vicinity of the point $v=0$, i.e. without any restrictions concerning the value of $v$. Besides, the proposed by us approach to the mathematical analysis of the LF behavior in contrast to the approach described in [12], has allowed us to develop the logically consistent method for the estimation of the statistical parameters of the task in the case when the second important parameter - a dispersion- is not known a priory. This is just the case which is most characteristic for the practice at solution of applied problems of the magnetic-resonance images processing.

The statements similar to the Theorem 1, has been obtained, in particular, in the mentioned paper [12]. In this paper a conclusion is made on the dependence of the LLF extremum's nature in the point $v=0$ upon the feasibility of the condition $\left\langle x^{2}\right\rangle>2 \sigma^{2}$. In the present paper we develop and apply the other approaches to the solution of this problem and we present here the mathematical consideration in detail because the similar logical constructions and the conclusions made on their basis will be used by us further, at grounding the method of the solution of two-parametric task In particular, by virtue of these reasons we provide here our proof of the Theorem 1, as subsequently the same statements will form the basis for the proof of the solution existence for two-parametric task.

\section{Two-Parametric ML Task: Theoretical Consideration}

We have considered above the mathematical task of the estimation of the statistical parameter $v$ based upon the data of a sample of $n$ measured random values of the signal's amplitude $x$. In this case the second parameter of the statistical model - a dispersion $\sigma^{2}$ - is supposed to be known a priori. However in fact this condition never takes place in practice what significantly decreases the merit and the accuracy of one parametric approach described above.

In this part of our paper we shall generalize the method for the case when both statistical parameters of the task $v$ and $\sigma^{2}$ are unknown. Consideration in this case generally demands a numerical solution of the system of two equations (4). In the present paper we have succeeded to reduce the problem to the numerical solution of one equation of one variable and so to simplify the task - both concerning the volume of calculations at the numerical computation and concerning the formal theoretical considerations and proofs.

Let us implement the change of one variable, namely: as two parameters of the task instead the variables $v$ and $\sigma^{2}$ we shall consider the following variables: $v$ and $\gamma=\frac{v}{\sigma^{2}}$. The introduction of the variable parameter $\gamma$ is purely formal technical trick that allows simplifying the subsequent calculations. A comprehensive mathematical analysis of the problem and strict substantiation of the maximum likelihood technique applicability for MR image processing tasks has become the subject of paper [21].

We'll obtain a system of ML equations for the introduced by us a pair of the statistical model's parameters $\nu$ and $\gamma$ by differentiating the LLF (3) by these parameters. From formula (1) we obtain a following representation of the probability density function $\hat{P}(x \mid v, \gamma)$ as a function of the parameters $v$ and $\gamma$, in the case when the signal's amplitude $x$ obeys to the Rice distribution:

$$
\widehat{P}(x \mid v, \gamma)=\frac{x \gamma}{v} \cdot \exp \left[-\frac{\gamma}{2} \cdot\left(\frac{x^{2}}{v^{2}}+1\right)\right] \cdot I_{0}(x \gamma)
$$

Let us consider a sample of $n$ measurements of the signal's amplitude value $x$. Then the $\operatorname{LF} \hat{L}(v, \gamma)$ is expressed as a product of the probability density functions for each measurement of this sample:

$$
\widehat{L}(v, \gamma)=\prod_{i=1}^{n} \widehat{P}\left(x_{i} \mid v, \gamma\right)
$$

Taking into account the formulas (9) and (10), we obtain for the LLF the following expression:

$$
\begin{aligned}
& \ln \widehat{L}(\nu, \gamma)=\sum_{i=1}^{n} \ln \widehat{P}\left(x_{i} \mid v, \gamma\right)= \\
& \sum_{i=1}^{n}\left\{\ln x_{i}+\ln \gamma-\ln v-\frac{\gamma \cdot v}{2}\left(1+\frac{x_{i}^{2}}{v^{2}}\right)+\ln I_{0}\left(x_{i} \cdot \gamma\right)\right\}
\end{aligned}
$$

The system of the likelihood equations for the parameters $v$ and $\gamma$ is as follows:

$$
\left\{\begin{array}{l}
\frac{\partial}{\partial v} \ln \widehat{L}(v, \gamma)=0 \\
\frac{\partial}{\partial \gamma} \ln \widehat{L}(v, \gamma)=0
\end{array}\right.
$$

The first of these equations has been discussed above at considering the one-parametric task (see equation (8)). In variables $v$ and $\gamma$ this equation looks as follows:

$$
v=\frac{1}{n} \sum_{i=1}^{n} \tilde{I}\left(x_{i} \gamma\right) \cdot x_{i}
$$

where the function $\tilde{I}\left(x_{i} \gamma\right)$ is determined by the formula (7). Differentiating the LLF (11) by variable $\gamma$, we obtain the second equation of the system (12) in the following view: 


$$
\frac{\partial}{\partial \gamma} \ln \widehat{L}(\nu, \gamma)=\frac{n}{\gamma}-\sum_{i=1}^{n} \frac{v}{2} \cdot\left(1+\frac{x_{i}^{2}}{v^{2}}\right)+\sum_{i=1}^{n} x_{i} \tilde{I}\left(x_{i} \gamma\right)=0
$$

Let us introduce a notation:

$$
S(\gamma)=\frac{1}{n} \sum_{i=1}^{n} x_{i} \tilde{I}\left(x_{i} \gamma\right)
$$

Then having conducted the non-complicated mathematical transformations we'll obtain the following system of equations for the parameters $v$ and $\gamma$ :

$$
\left\{\begin{array}{l}
v=S(\gamma) \\
\gamma=\frac{2 v}{\left\langle x^{2}\right\rangle+v^{2}-2 v S(\gamma)}
\end{array}\right.
$$

Substituting the first equation of the system (13) into the second one, we obtain the equation for the variable $\gamma$ :

$$
\gamma=\frac{2 S(\gamma)}{\left\langle x^{2}\right\rangle-S^{2}(\gamma)}
$$

It is evident that the issue on the existence and uniqueness of the solution of equation (14) means the existence and uniqueness of the solution of equations system (13). We have studied the properties of equation (14) solution both by means of computer simulation and by analytical mathematical considerations. The main conclusions of this study are the following:

1. The solution of equation (14) for non-negative parameter exists;

2. This solution is unique;

3. This solution corresponds to the global maximum of the LLF (11).

Now let us consider these assertions in more detail. The function is a linear combination of the function, and this determines the properties of the function as a smooth monotonous and concave function. We shall use these properties at investigating the issues concerning the existence and the properties of the solution of equation (14) for. The rather complicated analytical considerations having been implemented by us can be resumed as the following mathematical affirmation:

Theorem 2

The solution of the equation (14) for non-negative values of the parameter exists.

Unfortunately, the strict proof of the uniqueness of this solution, which is needed for the completely rigorous treatment of the problem, is unavailable now. Thus, let us declare this assertion as a hypothesis: The solution of the equation (14) for non-negative values of the parameter is unique.

The strict proof of this assertion has seemed to be a very cumbersome task, because the right part of equation (14) is neither a concave, nor a convex function at . Nevertheless we have got a lot of confirmations of the mentioned solution's uniqueness by virtue of the experimental results. The example plots of the LF shape presented in Fig. 1 for various signal-to-noise ratio values may illustrate the uniqueness of the two-parametric ML equations' solution. Based upon the assumption, that the two-parametric ML equations' solution is unique, we proved the following assertion:

Theorem 3

The unique non-zero solution of the equation (14) always corresponds to the global maximum of the LLF (11).

This conclusion may seem to be unexpected as from Theorem 1 it follows that the ML estimation may take the place at $v=0$ (what in our case would correspond to zero value of variable $\gamma$ and uncertain value of $\sigma$ ). Nevertheless this paradox is only the seeming one and may be explained as follows. The zero estimation of variable $v$ is possible only at a priory known value of $\sigma$ (let us denote it as $\sigma_{A}$ ). However in our case we use the value of $\sigma$ having been obtained by means of solving the maximum likelihood equation (we shall denote it as $\sigma_{M L}$ ). At such a value of $\sigma$ the mean value parameter $v$ can be infinitely close to zero, but cannot be equal strictly to zero.

\section{Two-Parametric ML Problem: Practical Issues}

The numerical solution of the equation (14) allows finding the value of the parameter $\gamma=\frac{v}{\sigma^{2}}$, and then, taking into account expression (13), determining the values of the parameters $v$ and $\sigma$ as well.

It is worth mentioning, that the proposed solution of the system (13) demonstrates a new meaning of the "conventional" estimation of the parameter $v$, provided by the formula

$$
v=\sqrt{\left\langle x^{2}\right\rangle-2 \sigma_{A}^{2}}
$$

where $\sigma_{A}^{2}$ is some hypothetic value specified a priori. In the paper [11] this formula was subjected to some just criticism due to the following issues:

1. It loses the sense at $\left\langle x^{2}\right\rangle<2 \sigma_{A}^{2}$;

2. It does not provide a strict estimation of the parameter from the viewpoint of the ML principle.

However, if we substitute the first equation of the system (13) into the second one with replacing the function $S$ by $v$ and the parameter $\gamma$ by $v / \sigma^{2}$, we obtain the following expression coinciding with the conventional estimation formula:

$$
v=\sqrt{\left\langle x^{2}\right\rangle-2 \sigma_{M L}^{2}}
$$


where $\sigma_{M L}$ means the estimation of the parameter $\sigma$, having been obtained as a result of the solution of the equations' system (13), i. e. corresponding to the maximum of the LF. Despite the fact that this formula looks exactly as a conventional one, one can affirm that just this formula provides the ML estimation for the parameter $v$ if the parameter $\sigma$ was not taken as a priory known $\sigma_{A}$ but was calculated by means of the ML equations' system (13) solving.

So, from the above analysis the conclusion follows that the system (13) always has a nontrivial solution and so the situation $\left\langle x^{2}\right\rangle<2 \sigma_{M L}^{2}$ never takes place. The calculation by the formula (16) always provides the ML estimation. Subsequently, we obtain the following optimal strategy of this method application:

1. By means of the numerical solution of the equations' system (13) a parameter $\sigma_{M L}^{2}$ is calculated for some homogeneous area of an image;

2. All other calculation are conducted by the formula (16) and do not demand a numerical solution of any equations.

The elaborated procedure allows accurate ML estimating of the statistical parameters of an image, based upon the measured data instead of any a priory assumptions. The obtained values of the parameters correspond to the maximum of the LF. This technique is not associated with cumbersome computing as the principle calculations are implemented by means of a simple formula (16).

\section{The Numerical Simulation Results}

The both above described ML approaches were tested by means of computer simulation (one- and two-parametric). The first numerical experiment was conducted as follows.
The data were generated obeying to the Rice distribution with the parameters given beforehand. Parameter $\sigma$ was assumed to be equal to 1 , while parameter $v$ was changed from 0 to 5 . So created sets were used to make measures of the parameters according to the above presented algorithms. The experiments were performed at variable sample's length $n$ : from 4 up to 64 . The same experiment for each value of $v, \sigma$ and $n$ was repeated $10^{4}$ times to acquire statistics.

For each $n$ four types of measurements were implemented:

1. Calculation of parameter $v$ by means of the ML method:

- traditional one-parametric ML estimation of $v$ by means of solving (8) assuming that parameter $\sigma$ is known a priory;

- original two-parametric ML estimation according to (13) for the case when both statistical parameters are unknown.

These results are presented by an upper row of the graphs in Fig. 2.

2. Calculation of the mean square deviation of the measured values of parameter $v$ for both methods. These results are presented by the second from top row of the graphs in Fig. 2.

3. Calculation of parameter $\sigma$ by means of the ML method: only two-parametric estimation according the equations (13). The results are presented by the third from top row of graphs in Fig. 2.

4. Definition of the mean square deviation of the measured values of parameter $\sigma$. These results are presented by lower row of the graphs in Fig. 2.

On the basis of the presented graphs were have investigated the errors inherent to both one-parametric and two-parametric methods. The results are compiles in Table 1

Table 1. Investigated methods' errors estimation

\begin{tabular}{|c|c|c|c|c|c|c|}
\hline Parameter & Error type, measurement method & $\mathrm{n}=4$ & $\mathrm{n}=\mathbf{8}$ & $\mathrm{n}=16$ & $\mathrm{n}=\mathbf{3 2}$ & $n=64$ \\
\hline \multirow{4}{*}{$v$} & Maximum systematic bias, one-parametric method ( $\sigma$ is known a priory) & 0,4 & 0,35 & 0,3 & 0,25 & 0,2 \\
\hline & Maximum systematic bias, two-parametric method ( $\sigma$ is found from the sample) & 0,9 & 0,8 & 0,7 & 0,6 & 0,5 \\
\hline & Maximum mean square deviation, one-parametric method ( $\sigma$ is known a priory) & 0,68 & 0,63 & 0,58 & 0,53 & 0,49 \\
\hline & Maximum mean square deviation, two-parametric method ( $\sigma$ is found from the sample) & 0,7 & 0,53 & 0,42 & 0,35 & 0,18 \\
\hline \multirow{4}{*}{$\sigma$} & Maximum systematic bias at small $v$ & $-0,4$ & $-0,39$ & $-0,21$ & $-0,16$ & $-0,12$ \\
\hline & Asymptotic systematic bias of $\sigma$ estimation at large $v$ & $-0,2$ & $-0,1$ & $-0,04$ & $-0,02$ & $-0,01$ \\
\hline & Maximum mean square deviation at small $v$ & 0,4 & 0,33 & 0,26 & 0,21 & 0,17 \\
\hline & Asymptotic mean square deviation at large $v$ & 0,3 & 0,25 & 0,18 & 0,13 & 0,09 \\
\hline
\end{tabular}



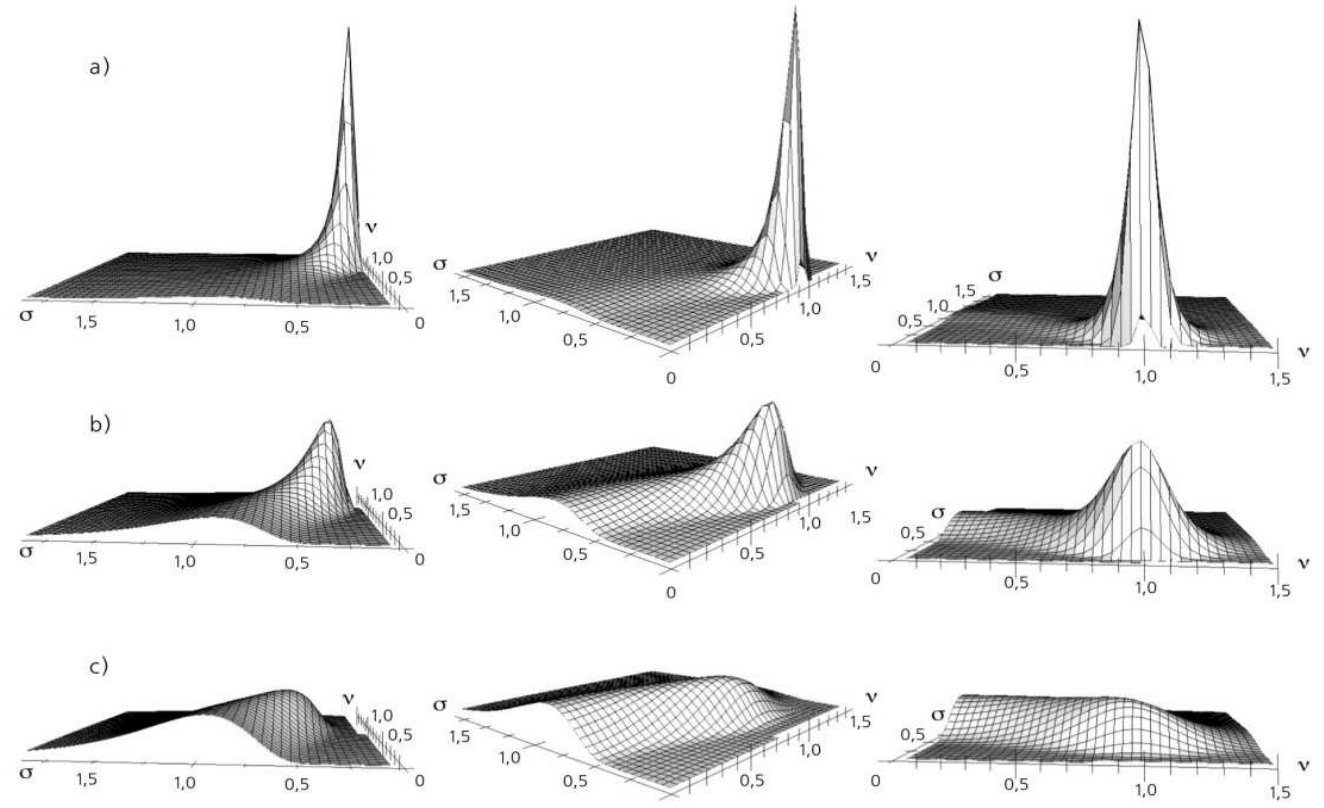

Fig. 1. The three-dimensional shape of the LF at various statistical parameters' values: a) $\boldsymbol{v}=1, \boldsymbol{Z}=, 1 ; b) \quad \boldsymbol{v}=1, \boldsymbol{\gamma}=, 25$; c) $\boldsymbol{V}=1, \boldsymbol{\sigma}=, 5$. Each plot is represented from 3 view angles: view from $\boldsymbol{\sigma}$ axis side (left column); view from the origin of the coordinates side (middle column); view from $\boldsymbol{V}$ axis side (right column). The sample length $n$ in these experiments equals to 3 .

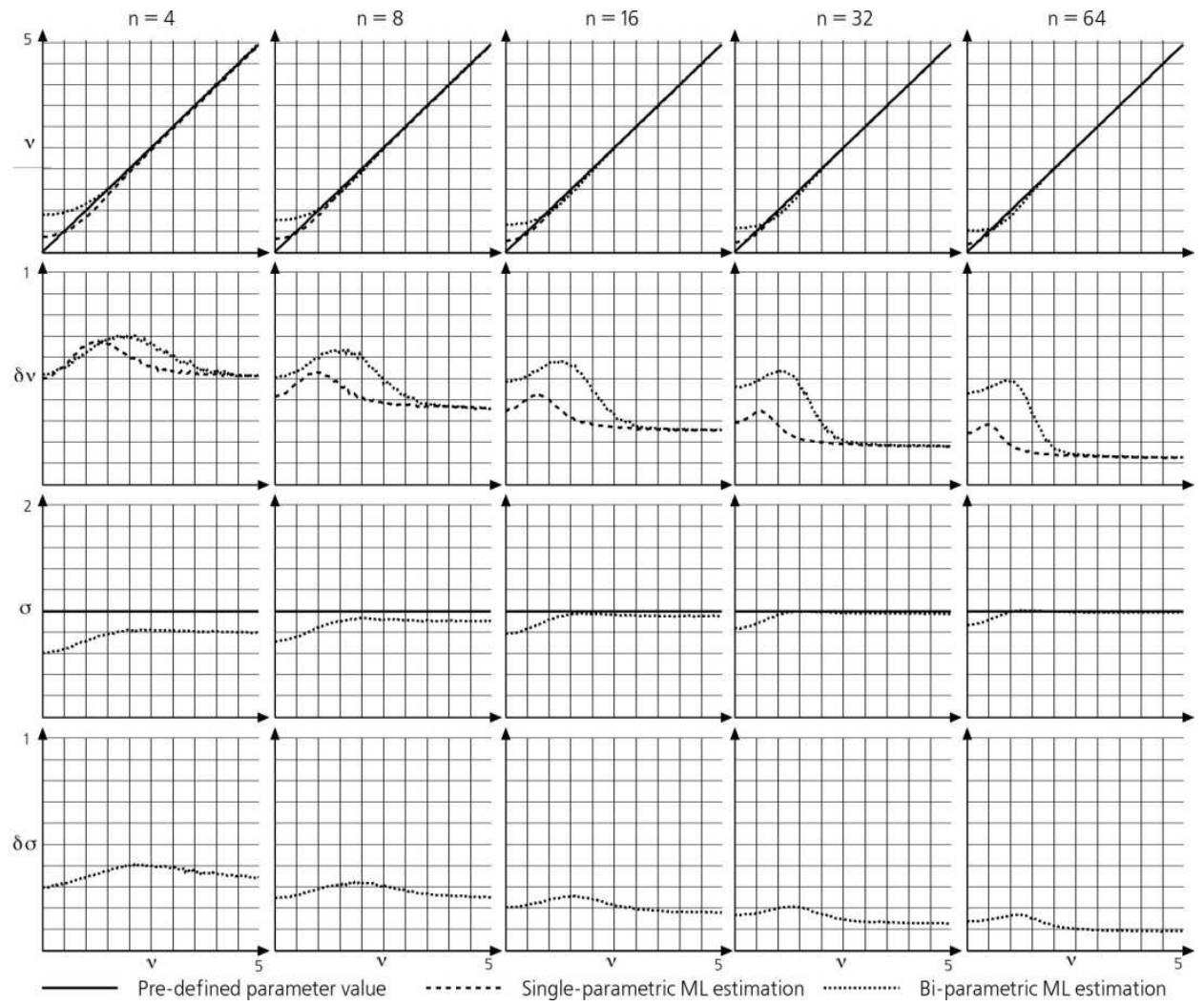

Fig. 2 Results of computer simulation. Along abscissa axis in all graphs a given value of $\boldsymbol{V}$ is marked, along ordinate axis a calculated value of variable parameter $\boldsymbol{V}$ is marked, the grid spacing equals to 0,5, the range of values: $0 \ldots 5$. The graphs rows from top to bottom contain: (1) measurements of $\boldsymbol{V}$ by two different methods (the grip spacing along the abscissa axis is 0,5; the range of values: 0...5); (2) mean square deviation of $\boldsymbol{V}$ (the grid spacing along abscissa axis is 0,1; the range of values: $0 . .1$ ); (3) measurements of $\sigma$ (the grid spacing along abscissa axis is 0,2; the range of values $0 . .2$ ); (4) the mean square deviation of $\boldsymbol{\sigma}$ (the grid spacing along abscissa axis is 0,1 ; the range of values: $0 \ldots 1$ ). 
As it follows from the data of Table 1, with the growth of the amount of the data points in a sample an error at computing the statistical parameters decreases noticeably (especially the mean square deviation).

Table 1 and Fig. 2 make clear the important peculiarity of the Rician distribution: both bias and variance of the measured $v$ values grow strongly at low SNR, which is inherent to both variants of the examined ML estimation procedures. This can be explained with the aid of Fig. 1 plots: one can see, that the less is SNR, the less expressed is the maximum of LF. Nevertheless, this maximum never disappears completely.
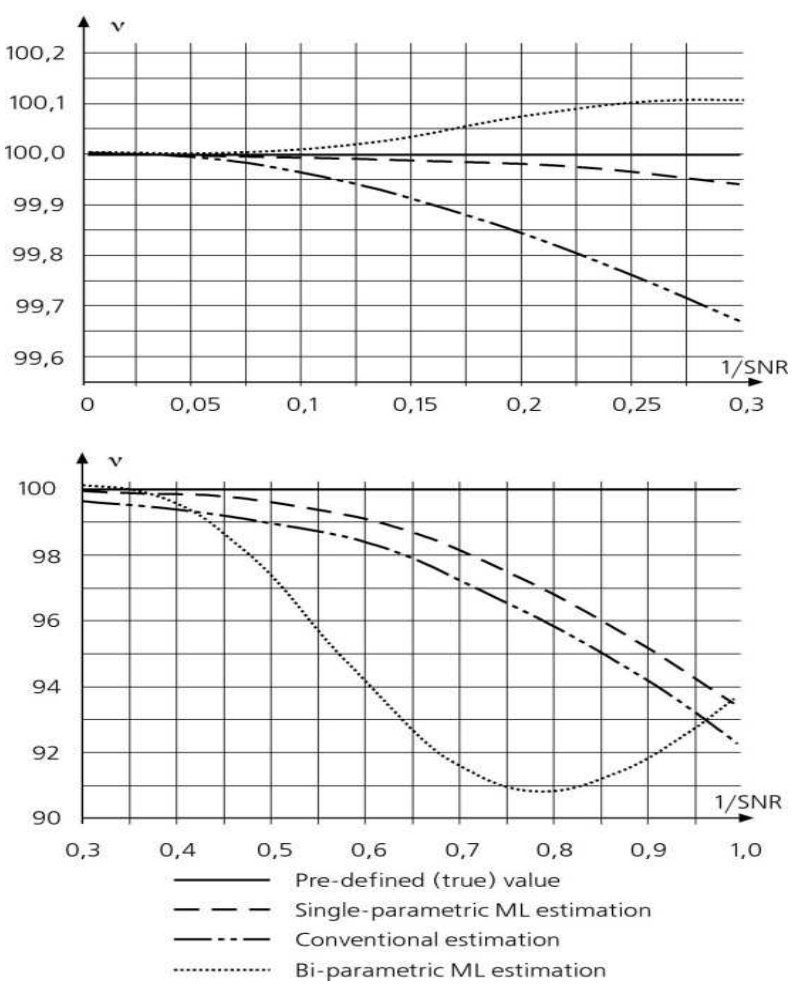

Fig.3. Comparing the obtained results with the results provided in [11].

While doing the numerical calculations of the ML solution for both unknown statistical parameters we found it reasonable to compare the obtained results with the results provided in paper [11] for the only unknown parameter in supposition of the a priory known parameter . Fig. 3 illustrates this comparison. The experiments in this case were performed in the same conditions as the analogous experiment in [11], so our Fig. 3 can be directly compared with the Fig. 2 in [11]. In this experimentthe simulated Rician distributed signal was estimated from 16 data points. The true value of $v$ was 100 , pre-assigned $\sigma$ values grow from 0 to 30 (upper graph), and from 30 to 100 in lower graph. The ML estimations were obtained by maximization of the LLF by numerical solving of the equations (8) and (13). Also there was done the conventional estimation by formula (15). The same experiment of $v$ estimation by three different ways was repeated $10^{5}$ times after which the average values were computed.
The bias in two-parametric ML estimation is evidently larger than in one-parametric $\mathrm{ML}$ and conventional estimations. This is easily explainable: $v$ estimation error in two-parametric task is influenced by the error of $\sigma$ estimation, while in one-parametric task $\sigma$ is known exactly. In this connection it is worth to note once more that the case of the only unknown parameter is not realistic in practice and so this comparison is rather provisional.

\section{Conclusion}

In the present paper a task has been solved consisting in the elaboration of a new so-called two-parametric technique for the estimation of unknown statistical parameters of a Rician distributed signal forming the magnetic-resonance image. This task is being solved by means of the ML method on the basis of measured sample's values of the signal according to the above-presented theoretical approach. The elaborated technique allows to obtain simultaneous estimation of both the noise and the signal unknown parameters of the Rice statistical distribution what opens new important perspectives in MR imaging development.

While providing significantly more possibilities for image processing if compared with the conventional one-parametric approach, the proposed two-parametric ML method has appeared to be approximately the same in its computational cost as the traditional one-parametric ML method.

At low values of the signal-to-noise ratio $(v<2 \sigma)$ the proposed here two-parametric method provides approximately 2 times worse accuracy of the estimation of parameter $v$ in compared with the traditional one-parametric ML method (when parameter $\sigma$ is assumed to be known a priory). This fact is easily explainable because the result of the traditional method is not influenced by an error of the parameter $\sigma$ estimation.

However, in its pure form the traditional method is not applicable in practice because the second statistical parameter $\sigma$ never may be considered as known a priori. At the same time at more high signal-to-noise ratios $(v>3 \sigma)$ the errors of the two-parametric method are practically indistinguishable from the errors inherent to traditional one-parametric method at the same sample length $n$. But for the case $v>3 \sigma$ one can affirm that the proposed method ensures practically the same accuracy as one-parametric method, at the same time it possess an essential advantage as for this method does not need any a priory information about the process.

The implemented analysis and the image processing method elaborated in the present paper allow reconstructing an image of the investigated object ensuring a correct suppression of non-linear Rician noise corruptions that appear in the systems of tomography visualization.

An important issue that should be noted here concerns the practical value of the results obtained in the present paper. The conducted mathematical research and the obtained experimental results have shown that the proposed 
two-parametric technique can be reliably applied for the image analysis tasks at rather high values of signal-to-noise ratios $(v>3 \sigma)$. We expect the possible opponents' objections that at high values of signal-to-noise ratios these is no need to use elaborated two-parametric approach to solve the tasks within the Rice statistical model as at this area the Gauss distribution can be applied. But such kind of objection would be not true due to the following reasons: the Gauss distribution starts properly working for the considered task at significantly higher values of signal-to-noise ratio, namely: at $v>10 \sigma$. So, there is a large interval of practically meaningful signal values $10 \sigma>v>3 \sigma$ in magnetic-resonance imaging which is adequately described by the Rician statistical model. And in this interval the elaborated two-parametric technique seems to be the most appropriate for describing and calculating the image processing tasks making use of all the advantages of this technique provided above.

In the conclusion it is worth to underline that the present paper provides a theoretical analysis of the maximum likelihood method for the two-parametric task in the Rician distributed image processing. The developed two-parametric version of the maximum likelihood method is a new effective tool both to process the Rician images and to evaluate the precision of other two-parametric techniques by comparing with the technique proposed in the present paper.

A principle merit of the elaborated technique consists in the proved possibility to obtain the unique values of both major statistical parameters of the image at the "cost" of one-parameter estimation.

\section{Appendix. Proofs of Principle Statements}

\section{Lemma 1}

Function $\tilde{I}(z)$ is positive-valued, monotonically increasing and concave at interval $(0,+\infty)$.

Proof:

The condition of monotonic character of the function $\tilde{I}(z)$ means the nonnegativity of its derivative. Taking into account the definition of the function $\tilde{I}(z)$ and the known formula $I_{0}^{\prime}(z)=I_{1}(z)$ we can put down its derivative as follows:

$$
\begin{aligned}
& \tilde{I}^{\prime}(z)=\frac{I_{1}^{\prime}(z) I_{0}(z)-I_{1}(z) I_{0}^{\prime}(z)}{I_{0}^{2}(z)} \\
& =\frac{I_{0}(z) \cdot I_{1}^{\prime}(z)-I_{1}^{2}(z)}{I_{0}^{2}(z)}
\end{aligned}
$$

The function $I_{0}$ in the denominator is always positive, so the sign of the derivative of function $\tilde{I}(z)$ is determined by the sign of (17) numerator. So to prove the monotonic character of the function $\tilde{I}(z)$, i.e. the nonnegativity of its derivative, it is sufficient to prove the nonnegativity of the numerator of (17) at $z \geq 0$.

Let us consider the known integral presentation of the modified Bessel function of the first kind of integer order:

$$
I_{n}(z)=\frac{1}{\pi} \int_{0}^{\pi} e^{z \cos \theta} \cos n \theta d \theta
$$

where $l \in \mathbf{Z}, \square(z)>\quad$. From this formula we can obtain the following expressions for functions $I_{0}(z)$ and $I_{1}(z)$ :

$$
I_{0}(z)=\frac{1}{\pi} \int_{0}^{\pi} e^{z \cos t} d t, I_{1}(z)=\frac{1}{\pi} \int_{0}^{\pi} e^{z \cos t} \cdot \cos t d t
$$

Similarly, for the derivative of the function $I_{1}(z)$ we obtain:

$$
I_{1}^{\prime}(z)=\frac{1}{\pi} \int_{0}^{\pi} e^{z \cos t} \cos ^{2} t d t
$$

Substituting (19) and (20) into the numerator of the expression (18) we get:

$$
\begin{gathered}
I_{1}^{\prime}(z) I_{0}(z)-I_{1}^{2}(z)= \\
\frac{1}{\pi^{2}} \int_{0}^{\pi} e^{z \cos t} \cos ^{2} t d t \cdot \int_{0}^{\pi} e^{z \cos t^{\prime}} d t^{\prime}- \\
-\frac{1}{\pi^{2}} \int_{0}^{\pi} e^{z \cos t} \cos t d t \cdot \int_{0}^{\pi} e^{z \cos t^{\prime}} \cos t^{\prime} d t^{\prime}
\end{gathered}
$$

As a result of non-complicated transforms we obtain for the expression (21) the following view:

$$
\begin{gathered}
I_{1}^{\prime}(z) \cdot I_{0}(z)-I_{1}^{2}(z)= \\
\frac{1}{2 \pi^{2}} \int_{0}^{\pi} \int_{0}^{\pi} d t d t^{\prime} e^{z\left(\cos t+\cos t^{\prime}\right)}\left(\cos t-\cos t^{\prime}\right)^{2}
\end{gathered}
$$

By virtue of nonnegative value of the sub-integral expression it obviously follows from (22) that the whole expression (22) is nonnegative and, consequently, the derivative of function $\tilde{I}(z)$ is nonnegative, what means its monotony at $z \geq 0$. So the function's monotony is proved.

The proof of concavity is implemented similarly, although it demands much more cumbersome calculations.

Lemma 2

$$
\forall z>0 \tilde{I}(z) \in(0,1)
$$

Proof: 
Let us consider the integral representation (18). At $n=0$ the sub-integral expression is always larger than the same expression at $n=1$. From this fact it easily follows that: $\forall z>0 I_{0}(z)>I_{1}(z)$. The lemma is proved.

Theorem 1

Let us suppose that the condition $\left\langle x^{2}\right\rangle>2 \sigma^{2}$ is valid. Then at $v>0$ there exists a unique solution of equation (8), which corresponds to the LF maximum. If $\left\langle x^{2}\right\rangle \leq 2 \sigma^{2}$, then the LF maximum corresponds to trivial solution of equation (8), i.e. to $v=0$.

Proof:

To determine the conditions of the existence of non-trivial solution of equation (8) let us consider the behavior of the right and the left parts of this equation.

The left part of equation (8) is presented by a straight line $y_{1}=v$. The right part, as it follows from the properties of function $\tilde{I}(z)$, having been proved in Lemma 1, is presented by a smooth monotonic concave function $y_{2}(v)=\frac{1}{n} \sum_{i=1}^{n} \tilde{I}\left(\frac{x_{i} v}{\sigma^{2}}\right) \cdot x_{i}$. The graph of this function is a curve passing through the coordinates origin point and asymptotically approaching to the straight line $y=\langle x\rangle$. Due to its concave character this curve can have one or two common points with the straight line $y_{1}=v$. One of these points is always known to us and corresponds to the trivial solution $v=0$.

The existence of the second common point is determined by the function $y_{2}(v)$ derivative in the vicinity of the coordinates' origin point: for the existence of the second solution it is necessary and sufficient that the condition $y_{2}^{\prime}(0)<y_{1}^{\prime}(0)=1$ takes place. Let us find the derivative $y_{2}^{\prime}(0)$ :

$$
y_{2}^{\prime}(v)=\frac{1}{n} \sum_{i=1}^{n} \frac{\partial}{\partial z_{i}} \tilde{I}\left(z_{i}\right) \cdot \frac{x_{i}^{2}}{\sigma^{2}}
$$

$z_{i}=\frac{x_{i} \nu}{\sigma^{2}}$ decomposition into a series [20]:

$$
I_{0}(z)=\sum_{k=0}^{\infty} \frac{(z / 2)^{2 k}}{k ! k !}, I_{1}(z)=\sum_{k=0}^{\infty} \frac{(z / 2)^{2 k+1}}{k ! \cdot(k+1) !}
$$

From decompositions (23) we obtain the following formula describing the behavior of function $\tilde{I}(z)$ at $z \rightarrow 0$ :

$$
\tilde{I}(z) \simeq \frac{z}{2} \cdot\left(1-\frac{z^{2}}{8}+O\left(z^{4}\right)\right)
$$

Taking into account this estimation of the function $\tilde{I}(z)$ at small values of argument, we obtain:

$$
y_{2}^{\prime}(0)=\frac{1}{n} \sum_{i=1}^{n} \frac{x_{i}^{2}}{2 \sigma^{2}}
$$

Thus, the non-trivial solution of equation (8) exists only at the condition $\left\langle x^{2}\right\rangle>2 \sigma^{2}$. To determine if any solution of equation (8) corresponds to maximum or to minimum of the LF we have to consider the second derivative of the LLF. From equation (5) taking into account expressions (6) and (7), we obtain:

$$
\frac{\partial^{2}}{\partial v^{2}} \ln L(v, \sigma)=y_{1}^{\prime}(v)-y_{2}^{\prime}(v)
$$

In the vicinity of zero this expression can be transferred into the following one:

$$
\frac{\partial^{2}}{\partial v^{2}} \ln L(v, \sigma)=1-\left\langle x^{2}\right\rangle / 2 \sigma^{2}
$$

So, at $\left\langle x^{2}\right\rangle>2 \sigma^{2}$ the second derivative in the vicinity of zero is positive what corresponds to the minimum of the LLF. Since the LLF and all its derivatives are smooth, its second extremum at $v>0$ can be only a maximum. At $\left\langle x^{2}\right\rangle<2 \sigma^{2}$ the second derivative in the vicinity of zero is negative and this means that the LLF has a maximum. So the theorem is proved.

Theorem 2

The solution of the equation (14) for non-negative values of the parameter $\gamma$ exists.

Proof:

Let us consider equation (14) at $\gamma \geq 0$, what corresponds to the physical sense of the task. Then the left part of equation (14) is presented by a straight line $y_{3}(\gamma)=\gamma$, passing through the coordinates' origin point. Let us consider the behavior of the equation's right part:

$$
y_{4}(\gamma)=\frac{2 S(\gamma)}{\left\langle x^{2}\right\rangle-S^{2}(\gamma)}
$$

The denominator of this expression is positive monotonically decreasing function by virtue of lemmas 1 and 2 while the nominator is a positive monotonically increasing function. So the function itself is monotonically increasing.

Taking into account the series decomposition formulas (23), we obtain the following expression, which is valid at vanishing values of variable $\gamma$ :

$$
\lim _{\gamma \rightarrow 0} y_{4}(\gamma)=\gamma \cdot\left(1+\gamma^{2} \frac{\left\langle x^{2}\right\rangle}{4}\right)>\gamma
$$


Thus in the vicinity of zero value of variable $\gamma$ the curve $y_{4}(\gamma)$, displaying the right part of equation (14), goes above the straight line corresponding to the left part of this equation: $y_{3}(\gamma)=\gamma$.

At larger values of variable $\gamma$ the right part of equation (14), by virtue of the asymptotic behavior of the modified Bessel functions, can be put down as follows:

$$
\lim _{\gamma \rightarrow \infty} y_{4}(\gamma)=\frac{2\langle x\rangle}{\left\langle x^{2}\right\rangle-\langle x\rangle^{2}}>0
$$

It is easily seen that the denominator of expression (25) is a non-negative magnitude and equals to mean squared deviation of a sample value $x_{i}$. So with increasing the value of variable $\gamma$ the right part of equation (14) asymptotically approaches to the constant positive value determined by expression (25). This means that the curve corresponding to the right part of equation (14) inevitably crosses the straight line corresponding to the left part of this equation because in the vicinity of zero this curve goes above the mentioned straight line. In other words, from the above considerations we can conclude that the non-trivial solution of equation (14) exists. The theorem is proved.

Theorem 3

The unique solution of the equation (14) corresponds to the global maximum of the LLF (11).

Proof:

Let us find the second derivative of the $\ln \widehat{L}(v, \gamma)$ function with respect to $\gamma$ :

$$
\begin{aligned}
& \frac{1}{n} \frac{\partial^{2}}{\partial \gamma^{2}} \ln \widehat{L}(v, \gamma)=\left(\frac{1}{\gamma}+\frac{S(\gamma)}{2}-\frac{\left\langle x^{2}\right\rangle}{2 S(\gamma)}\right)^{\prime} \\
& =-\frac{1}{\gamma^{2}}+\frac{S^{\prime}}{2}+\frac{S^{\prime}}{2 S^{2}}\left\langle x^{2}\right\rangle
\end{aligned}
$$

Making use of expressions having been obtained by us while proving the Theorem 1, we get the following estimation at $\gamma \rightarrow 0$ :

$$
\lim _{\gamma \rightarrow 0} \frac{1}{n} \frac{\partial^{2}}{\partial \gamma^{2}} \ln \hat{L}(\nu, \gamma)=\frac{\left\langle x^{2}\right\rangle}{4}+\frac{1}{16} \frac{\left\langle x^{3}\right\rangle}{\left\langle x^{2}\right\rangle},
$$

This means that the second derivative of the LLF is always positive at zero point and consequently this point corresponds to its minimum. As the LLF is smooth, its second extreme point can be only a maximum.

\section{References}

[1] T. Wang and T. Lei, "Statistical analysis of MR imaging and its application in image modeling," in Proc. IEEE Int. Conf.
Image Processing and Neural Networks, vol. I, 1994, pp. 866-870.

[2] S. O. Rice, "Mathematical analysis of random noise," Bell Syst. Technological J., vol. 23, p. 282, 1944.

[1] R. M. Henkelman, "Measurement of signal intensities in the presence of noise in MR images". Med. Phys., vol. 12, no. 2, pp. 232-233, 1985.

[2] A. Papoulis, Probability, Random Variables and Stochastic Processes, 2nd ed. Tokyo, Japan: McGraw-Hill, 1984.

[3] H. Gudbjartsson and S. Patz, "The Rician distribution of noisy MRI data”, Magn. Reson. Med., vol.34, pp.910 — 914, 1995.

[4] A. Macovski, "Noise in MRI”, Magn. Reson. Med., vol. 36, No.3, pp.494-497, 1996.

[5] G. Gerig, O. Kubler, R. Kikinis, and F. A. Jolesz, "Nonlinear anisotropic filtering of MRI data," IEEE Trans. Med. Imag., vol. 11, pp. 221-232, June 1992.

[6] G. Z. Yang, P. Burger, D. N. Firmin, and S. R. Underwood, "Structure adaptive anisotropic filtering for magnetic resonance image enhancement," in Proc. CAIP, pp. 384-391, 1995.

[7] S. J. Garnier and G. L. Bilbro, "Magnetic resonance image restoration,” J. Math. Imag., Vision, vol. 5, pp. 7—19, 1995.

[8] G. McGibney and M. R. Smith, "An unbiased signal-to-noise ratio measure for magnetic resonance images," Med. Phys., vol. 20, no. 4,pp. 1077-1078, 1993.

[9] Jan Sijbers, Arnold J. den Dekker, Paul Scheunders, and Dirk Van Dyck, "Maximum-Likelihood Estimation of Rician Distribution Parameters", IEEE Transactions on Medical Imaging, vol.17, No 3, p.p. 357-361, June 1998.

[10] Jeny Rajan, Ben Jeurissen, Marleen Verhoye, Johan Van Audekerke and Jan Sijbers, "Maximum likelihood estimation based denoising of magnetic resonance images using restricted local neighborhoods". Physics in Medicine and Biology, vol. 56, issue 16, pp. 2011. DOI: 10.1088/0031-9155/56/16/009

[11] Jeny Rajan, Dirk Poot, Jaber Juntu and Jan Sijbers, "Noise measurement from magnitude MRI using local estimates of variance and skewness", Phys. Med. Biol. 55, p.p.441-449, 2010.

[12] J. Sijbers \& A. J. den Dekker, "Maximum Likelihood estimation of signal amplitude and noise variance from MR data”. Magn Reson Med 51(3):586—594, 2004.

[13] L. He \& I. R. Greenshields , “A Nonlocal Maximum Likelihood Estimation Method for Rician Noise Reduction in MR images". IEEE Trans Med Imaging 28:165-172, 2009.

[14] Aja-Fernandez, S.; Alberola-Lopez, C.; Westin, C.-F. Noise and Signal Estimation in Magnitude MRI and Rician Distributed Images: A LMMSE Approach // IEEE Transactions on Image Processing, vol. 17, issue 8, pp. 1383-1398, 2008.

[15] C. F.M. Carobbi, M. Cati, "The absolute maximum of the likelihood function of the Rice distribution:existence and uniqueness, IEEE Trans. on Instrumentation and Measurement, vol 57, No 4, April 2008, pp. 682-689. 
[16] Rytov, S.M. Introduction into Statistical Radio-physics. P.1. Random processes. - Moscow: Nauka, 1976 (in Russian).

[17] P.J.Bickel, K.A.Doksum, Mathematical Statistics, Holden-Day, Inc., 1983.

[18] M. Abramowitz and I. Stegun (eds.) Handbook of Mathematical Functions with formulas, graphics and mathematical tables. National Bureau of Standards, applied mathematics series-55; Issued June 1964.

[19] T. Yakovleva. "Two-parametric method of noise and signal determination in magnetic resonance imaging: mathematical substantiation", unpublished.
[20] Young, Phillip M., et al. "MR imaging findings in 76 consecutive surgically proven cases of pericardial disease with CT and pathologic correlation." The international journal of cardiovascular imaging 28.5 (2012): 1099-1109.

[21] Samarin, Andrei, et al. "PET/MR imaging of bone lesions-implications for PET quantification from imperfect attenuation correction." European journal of nuclear medicine and molecular imaging 39.7 (2012): 1154-1160.

[22] Hualong Zu, Qixin Wang, Mingzhi Dong,Liwei Ma, Liang Yin, Yanhui Yang. "Compressed Sensing Based Fixed-Point DCT Image Encoding", Advances in Computational Mathematics and its Applications, 2.2 (2012): 259-262 